\title{
Cranial Ultrasonography in Evaluation of Meningitis in Neonates and Infants
}

\author{
Kalpeshkumar Patel ${ }^{1}$, Rajeshkumar Rathore ${ }^{2}$, C. Ray Chaudhuri ${ }^{3}$ \\ ${ }^{1}$ Associate Professor, Department of Radiology, ${ }^{2}$ Associate Professor, Department of Radiology, ${ }^{3}$ Professor \& Head, \\ Department of Radiology, S.B.K.S. Medical Institute \& Research Centre, India
}

Corresponding author: Dr. Rajeshkumar Rathore, Associate Professor, Department of Radiology, Dhiraj hospital, Piparia, Waghodia, Vadodara, Gujarat, India

DOI: http://dx.doi.org/10.21276/ijcmsr.2019.4.4.21

How to cite this article: Kalpeshkumar Patel, Rajeshkumar Rathore, C. Ray Chaudhuri. Cranial ultrasonography in evaluation of meningitis in neonates and infants. International Journal of Contemporary Medicine Surgery and Radiology. 2019;4(4):D87-D90.

\section{A B S T R A C T}

Introduction: Cranial ultrasonography plays an important role in the initial evaluation of infants with suspected meningitis and in evaluation of complications of the disease. Meningitis is a serious infection and commonly affects newborns, infants and children with grave consequences. There are many recent advances in diagnosis and treatment of meningitis. However, still there is residual neurological disability and morbidity. Study aimed to establish role of ultrasonography in evaluation of meningitis \& its complication in neonates \& infants.

Material and methods: 26 Patients with suspected case of meningitis with clinical findings of meningitis and/ or laboratory findings were performed cranial ultrasonography (Logiq P9 GE \&Logiq P5 GE) with linear probe (7 to $11 \mathrm{MHz}$ ) and Curvilinear probe ( 3 to $5 \mathrm{MHz}$ ).

Results: A study was performed on 26 patients (newborn to one year old) of suspected meningitis. Sonograms were obtained within 72 hours of birth, and repeated on 7th, 14th or at an early date, if required. 5 patients were normal on sonography. The spectrum of sonographic abnormalities includes echogenic sulci (53.8\%) and parenchyma (30.7\%), ventriculomegaly (46\%), ventriculitis (23\%), cerebral infarction (3.8\%), subdural fluid collection/ Subdural empyema (7.6\%), extradural fluid collection (3.8\%), encephalomalacia (3.8\%) and cerebral abscess $(7.6 \%)$ in patients.

Conclusion: Cranial ultrasonography continues as an excellent first line imaging investigation in suspected meningitis cases of infants for initial diagnosis, complications and monitoring the course of the disease. It is a reliable modality and provides useful information for diagnosis and treatment of meningitis and its complications.

Keywords: Meningitis, Ultrasonography, Doppler, Central Nervous System Infections, Neonates.

\section{INTRODUCTION}

Even with recent advances in antibiotic and supportive treatment, meningitis is still an important cause of newborn and infant mortality and morbidity. Early diagnosis and timely appropriate treatment of the complications can reduce the morbidity and mortality to a considerable extent. ${ }^{1}$

The most common cause of neonatal meningitis is bacterial infection. The most commonly isolated causative organisms in CSF of infants with meningitis are Group B streptococci, Escherichia coli and Listeria monocytogenes. Less common gram-negative enteric organisms include Klebsiella species and Citrobacter species. Delayed detection and treatment of neonatal meningitis may cause cerebral palsy, seizure disorder and learning deficiencies. ${ }^{2}$

The use of sonography to examine these neonates \& infants has rapidly increased in the last few years and the relative simplicity of the procedure, makes it an ideal method for early detection of complications and follow-up of these patients. Cranial sonography is mostly an initial imaging investigation for the evaluation of suspected meningitis in new borns and infants. With the help of Doppler ultrasound, one can differentiate benign enlargement of subarachnoid spaces from subdural effusions. ${ }^{3}$

Sonography is extremely beneficial in evaluating intraventricular contents, especially debris and intra-ventricular septations. $^{4}$

The objectives of study were early diagnosis of meningitis by cranial USG, early detection of complications of meningitis $\&$ early therapeutic intervention, establish the relationship between imaging findings and underlying pathological changes and establishment of cranial USG as $1^{\text {st }}$ line imaging modality of choice.

\section{MATERIAL AND METHODS}

The study was performed in the department of Radiodiagnosis, Dhiraj Hospital, S.B.K.S. Medical Institute and Research Centre, Pipariya, Vadodara. The study is Observational, Descriptive Hospital Based Study. Only those patients who were willing to participate in study were included. Patients who were suspected clinically and pathologically for meningitis were included in this study. 
Sample size of the study is 26 patients, out of which 5 patients were normal on imaging study.

The age of the patients in this study is newborn and infants up to 1 year. Out of 26 patients, 14 patients were male and 12 were female. Sonograms were obtained in newborn within 72 hours of birth, and repeated on 7th, 14th or at an early date, if required.

Cranial ultrasonography was performed on Logiq P9 GE and Logiq P5 GE sonography machines. Patients were examined with both the probes: Linear probe (7 to $11 \mathrm{MHz}$ ) and Curvilinear probe (3 to $5 \mathrm{MHz}$ ). Trans-fontanelle coronal, axial and sagittal images were taken. Sedative agents were used when needed e.g. in case of excessive crying. All aseptic precautions were taken to reduce any chances of infection to neonates and infants. Brain parenchyma, ventricular system, brain stem, cerebellar hemispheres, outline and content of ventricles, CSF space and extra-axial spaces were carefully examined. After cranial ultrasonography, clino-radiopathological correlation was done and patients were treated according to severity of the disease.

The patients were evaluated for presence of echogenic sulci, parenchymal echogenecity whether increased or decreased, size of the ventricles, presence of ventriculitis (echogenic ependyma, ventricular strands and echoes within the ventricles), any extraaxial fluid collection (e.g. subdural empyema) and for any evidence of abscess, infarcts or encephalomalacia.

\section{RESULTS}

26 patients of suspected meningitis were evaluated with cranial ultrasonography. Out of these 26 patients, 5 (19.33\%) patients were normal on ultrasonogrpahy despite clinical and biochemical profile of meningitis. $21(80.67 \%)$ patients were having imaging findings of meningitis. Out of 26 patients $14(53.84 \%)$ were male and 12 (46.16\%) were female, out of

\begin{tabular}{|l|c|c|c|}
\hline Diagnosis & Male & Female & Total \\
\hline Normal study & 3 & 2 & 5 \\
\hline Abnormal imaging findings & 11 & 10 & 21 \\
\hline Total & 14 & 12 & 26 \\
\hline Tabl: Radioleging
\end{tabular}

Table-1: Radiological findings according to gender distribution.

\begin{tabular}{|l|c|c|}
\hline Findings & Number & $\begin{array}{c}\text { Percentage } \\
\text { (\%) }\end{array}$ \\
\hline Normal & 5 & 19.2 \\
\hline Echogenic \& widened sulci & 14 & 53.8 \\
\hline Focal parenchymal opacities & 8 & 30.7 \\
\hline Ventriculomegaly & 12 & 46 \\
\hline $\begin{array}{l}\text { Ventriculitis } \\
\text { (Ependymitis, Choroid plexitis, Septa } \\
\text { formation, Ventricular exudates) }\end{array}$ & 6 & 23 \\
\hline Subdural empyema, Subdural effusion & 2 & 7.6 \\
\hline Epidural effusion & 1 & 3.8 \\
\hline Abscess formation & 2 & 7.6 \\
\hline Encephalomalcia & 1 & 3.8 \\
\hline Communicating Hydrocephalus & 8 & 30.7 \\
\hline Haemorrhagic infarcts & 1 & 3.8 \\
\hline \multicolumn{2}{|c|}{ Table-2: Sonographic spectrum in cases of meningitis (N=26) } \\
\hline
\end{tabular}

them $3(11.53 \%)$ males and $2(7.69 \%)$ females are normal on imaging study. (Table 1)

The most common imaging finding is echogenic sulci and widening of sulci. It was seen in $14(53.8 \%)$ patients. There were $8(30.7 \%)$ patients having focal areas of increased echogenecity of brain parenchyma and out these patients,

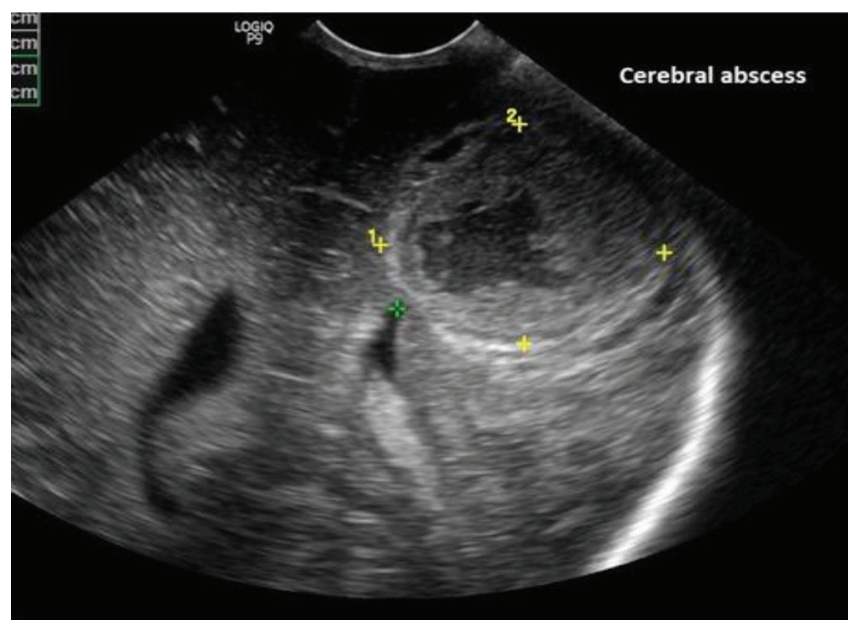

Figure-1: Sonographic image of neonatal brain shows well defined round lesion with smooth margins and heterogeneous content suggestive of cerebral abscess.
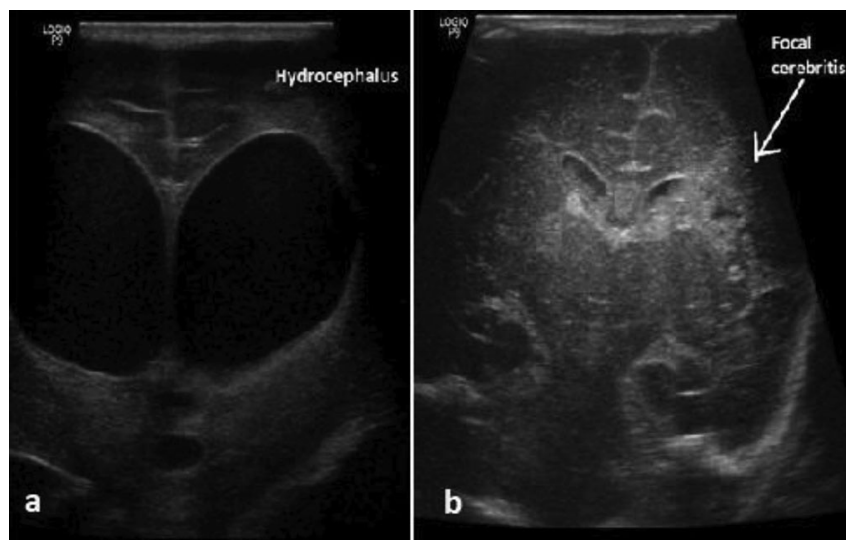

Figure-2: Transcranial ultrasonography in a patient shows (a) ventriculomegaly involving bilateral frontal horn \&(b) changes of focal cerebritis in periventricular region.
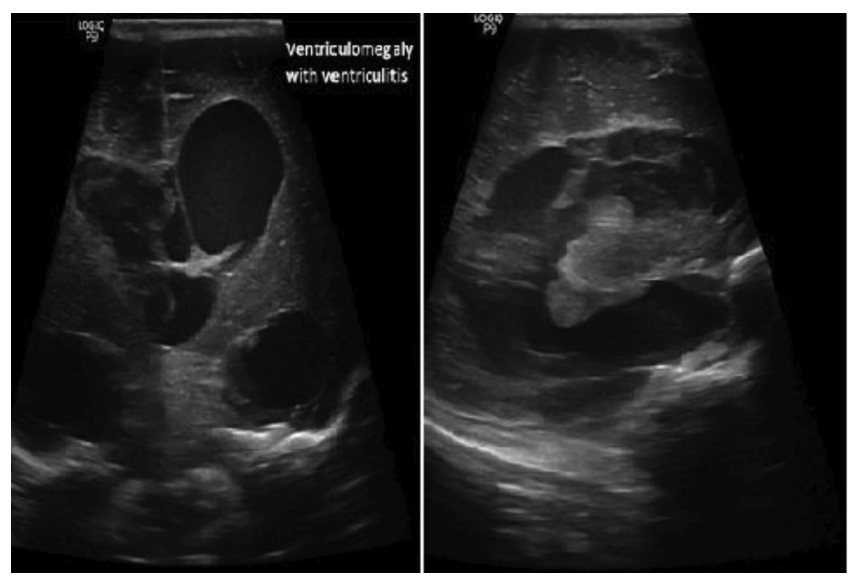

Figure-3: Axial and sagittal images show ventriculomegaly with exudates, debris with septations \& thickening of ependyma suggestive of ventriculitis. 


\begin{tabular}{|l|c|c|c|}
\hline Follow up Day & $\begin{array}{c}\text { Echogenic } \\
\text { sulci and } \\
\text { widening } \\
\text { (Out of 5 } \\
\text { patients) }\end{array}$ & $\begin{array}{c}\text { Parenchymal } \\
\text { opacities } \\
\text { (Out of 4 pts) }\end{array}$ & $\begin{array}{c}\text { Ventriculomeg- } \\
\text { aly and abscess } \\
\text { formation } \\
\text { (Out of 7 pts) }\end{array}$ \\
\hline $7^{\text {th }}$ day & 2 & 2 & 7 \\
\hline $14^{\text {th }}$ day & 1 & 2 & 7 \\
\hline $\begin{array}{l}\text { On date of } \\
\text { discharge }\end{array}$ & 1 & 1 & 7 \\
\hline \multicolumn{3}{|c|}{ Table-3: Follow up Imaging findings } \\
\hline
\end{tabular}

$2(7.6 \%)$ patients developed cerebral abscess formation in subsequent scans. The sonographic appearance of Abscess was well-circumscribed, complex cystic mass with highly echogenic walls and marked peripheral hyperaemia. (Figure 1)

Ventriculomegaly was observed in 12 patients (46\%); out of them 6 patients (23\%) have imaging findings of ventriculitis. Ventriculitis was seen in form of echogenic irregular ependyma, choroid plexitis, septae and ventricular exudates. 8 patients (30.7\%) were found having communicating hydrocephalus.(Figure 2 and Figure 3)

Subdural empyema, epidural and subdural effusions were observed in 3 patients (11.4\%). Haemorrhagic infarcts were found in 1 patient (3.8\%) and 1 patient (3.8\%) was found having imaging findings of encephalomalcia on follow up images. (Table 2)

Out of 26 patients, 16 patients (61.53\%) were followed up; it was observed that out of these 16 patients who were diagnosed on initial features of meningitis (Sulcal hyperechogenecity and widening) and were given timely antibiotic treatment, there was remarkable improvement in imaging. The patients who developed ventriculomegaly and other complications were found having same imaging findings on subsequent sonograms (Table 3).

\section{DISCUSSION}

In the pediatric age group, infective meningitis is an important and common cause of mortality and long term morbidity. There is a high mortality in acute stages and serious neurological sequelae in the survivors. An early identification of macroscopic pathological changes is important for timely diagnosis and treatment.

In the early stages, first, there is widening and increased echogenicty of sulci on cranial ultrasonography due to intense inflammatory exudate accumulating in the fissures and sulci, particularly around pia and subarachnoid vessels. In our study it was observed in $53.8 \%$ neonates and infants where as Han et al. reported it $82 \%$ cases. ${ }^{5}$ J.P. Soni did a study and found that echogenic sulci as the earliest finding in case of meningitis. ${ }^{6}$

Abnormal parenchymal echoes, diffuse or localized represents cerebritis or infarction, common in complicated meningitis carrying poor prognosis as reported earlier. Increased parenchymal echogenicity with chinked, small size ventricles suggested cerebral oedema and this required prolonged decongestive therapy. In our study it was found in $30.7 \%$ patients. Our study is consistant with Chowdharyet al. reported this in $65 \%{ }^{7}$
Ventriculomegaly can be an early or late finding on sonography. An early ventriculomegaly represents non-obstructive normal pressure hydrocephalus and is usually reversible. However, late ventriculomegaly is because of obstruction secondary to accumulation of purulent exudates (acute) or secondary to chronic inflammatory changes within ventricles or in the subarachnoid space. In our study it was found in $46 \%$ patients. D Baruah et al. found ventriculomegaly in $28.6 \%$ patients. ${ }^{8}$ This finding is consistent with other studies. ${ }^{9}$

Ventriculitis usually arises from the choroid plexus. On sonography, there is echogenic irregular ependyma with irregular hyperechoic choroid plexus and ventricular exudates. If the diseases progresses, after 2-3 weeks sonography shows formation of septations and compartments in the ventricles. In the present study, ventriculitis was observed in $23 \%$ cases in the form of ependymitis, choroid plexitis, ventricular exudate and septae.

Abscess is seen as sharply circumscribed lesion with thick rim of intense echogenicity surrounding the hypoechoic centre. The abscess is usually found in an area that previously exhibit increased echogenicity and poor margination, representing a focus of cerebritis, vasculitis or parenchymal infarction that becomes infected. In our study it was reported in 7.6\% patients. Subdural and extradural effusion and subdural empyema were reported as complication of meningitis. ${ }^{10}$

\section{CONCLUSION}

Cranial ultrasonography in newborns and infants is a rapid, safe and effective method for initial diagnosis, to identify complications and plan treatment of meningitis, even when signs and symptoms are vague or nonspecific. Ultrasound gives information regarding early and late changes seen in meningitis. Therefore, appropriate medical treatment can be timely initiated and monitored by sequential sonography and appropriate surgical intervention can be done when indicated.

\section{REFERENCES}

1. Fisher RM, Lipinski JK, Cremin BJ. Ultrasonic assessment of infectious meningitis. Clin Radio 1984; 35(2): 257-273.

2. Tunkel AR, Hartman BJ, Kaplan SL et al. Practice guidelines for the management of bacterial meningitis. Clin Infect Dis 2004;39(4):1267-1284

3. A. Yikilmaz, G. Taylor. Sonographic findings in bacterial meningitis in neonates and young infants. Pediatr Radiol 2008;38(6):129-137

4. N. Gupta, H. Grover, I. Bansal, K. Hooda, J. Sapire, R. Anand, Y. Kumar. Neonatal cranial sonography: ultrasound findings in neonatal meningitis- a pictoral review. Quant Imaging Med Surg 2017; 7(1): 123-131.

5. Han BK, Babcock DS, Me Adams L. Bacterial meningitis in infants: Sonographic findings. Radiology 1985;154(4): 645-650.

6. J. P. Soni, B.D. Gupta, M. Soni, M. Gupta, D.R. Dabi, K.R. Nemal. Cranial ultrasonic assessment of infants with acute bacterial meningitis. Indian Pediatrics 1994; 31(4):1337-1343

7. Chowdhary V, Gulati P, Sachdcv A, Mittal SK. Pyogenic meningitis: Sonographic evaluation. Indian Pediatr 
1991;28(6): 749-755.

8. D Baruah, N Gogoi, RK Gogoi. Ultrasound evaluation of acute bacterial meningitis \& its sequale in infants. Ind J Radiol Imag 2006;16:4: 553-558

9. Bhat V. Neonatal neurosonography: A pictoral essay. Indian J Radiol Imaging 2014: 24:389-400

10. Kastrup O, Wanke I, Maschke M. Neuro-imaging of infections. Neuro Rx 2005;2(5):324-332

Source of Support: Nil; Conflict of Interest: None

Submitted: 12-09-2019; Accepted: 30-10-2019; Published online: 24-11-2019 